\title{
The curative effects of methylsulfonylmethane against glycerol- induced acute renal failure in rats
}

\author{
Shaza Anwar Al laham \\ Department of Pharmacology \& Toxicology, Faculty of Pharmacy, Damascus University, Syria
}

\begin{abstract}
The present study was performed to explore the curative effect of Methylsulfonylmethane (MSM) in an experimental model of myoglobinuric acute renal failure (ARF). In this experimental model, Rats were injected with $50 \%$ glycerol $(10 \mathrm{~mL} / \mathrm{kg}$, im) followed by an hour later and daily in the next six days by MSM (400 mg/kg) or saline. Kidney's function (urea and creatinine), and reduced glutathione were analyzed. A renal failure produced by glycerol injection, with a significant increase of blood urea and serum creatinine was observed. Rats that received MSM in addition to glycerol had significantly lower blood urea and serum creatinine levels compared to those receiving glycerol alone. However, glutathione has markedly increased after MSM treatment. The effect is probably due to the antioxidant activity of MSM. This may provide therapeutic opportunities for treating humans, myoglobinuric ARF.
\end{abstract}

Keywords: Methylsulfonylmethane (MSM)/curative effects. Acute renal failure. Myoglobinuria. Reactive oxygen species.

\section{INTRODUCTION}

Methyl Sulphonyl Methane, better known as MSM, is a naturally occurring Sulphur compound with well-known antioxidant properties (Marañón et al., 2008). MSM may be effective for the treatment of pain syndromes, athletic injuries, bladder disorders, hyperacidity, constipation, musculoskeletal pain, allergies, and for immunomodulation (Parcell, 2002), osteoarthritis pain, inflammation, repetitive stress injuries, induce wound healing, bladder disorders like intestinal cystitis (Childs, 1994), parasitic infections and the maintenance of normal keratin levels in hair, skin and nails (Horvath et al., 2002). MSM may be used as a precursor for the synthesis of methionine and cysteine, sulfur-containing amino acids, and act as a source of sulfur (Bohlooli et al., 2013). Oral use of MSM has mild adverse effects, such as gastrointestinal (GI) symptoms, headaches, amplified effects of blood-thinning drugs resulting in easy bruising and blood in stool, increased blood pressure, increased hepatic enzymes, and insomnia if took at bedtime (Kim et al., 2006).

Correspondence: S. A. Al laham. Department of Pharmacology \& Toxicology, Faculty of Pharmacy, Damascus University, Syria, Damascus UniversityDamascus-Syria. Phone: 009630933375655. Email: lahamshaza@gmail.com
Acute renal failure (ARF) is a syndrome distinguished by an acute loss of renal function. Mortality from ARF remains high (over 50\%), despite the reversibility of this loss in most patients who survive. Therefore, the search for effective therapy to accelerate recovery and attempts to prevent ARF has attracted much attention (Park et al., 2012).

Acute renal failure (ARF) is characterized by a rapid, potentially reversible, decline in renal function including rapid fall in glomerular filtration rate (GFR), and retention of nitrogenous waste products over a period of hours or days. Rhabdomyolysis is the syndrome characterized by breakdown of striated muscle with a massive release of myoglobulin into the extracellular fluid and circulation leading to filtration of myoglobulin to renal tubules (Vanholder et al., 2000), which forms obstructing tubular casts, thus Rhabdomyolysis provokes acute tubular necrosis (ATN) (Singh et al., 2012).

The two critical factors that predispose to myohemoglobinuric ARF: hypovolemia/dehydration and aciduria. It is demonstrated that in their absence, hemeproteins have minimal nephrotoxic effects. At the nephron level, three basic mechanisms underlie hemeprotein toxicity: renal vasoconstriction, and direct hemeprotein-induced cytotoxicity (Zager, 1996).

Glutathione $(\mathrm{GSH})$ is an important tripeptide 
thiol ( $\gamma$-glutamyl cysteinyl glycine) antioxidant and its intracellular concentration is an indicator of oxidative stress, which has a profound effect on the cellular thiol balance and can lead to a decreased GSH/GSSG ratio in many body organs. Oxidative stress is a fact of many diseases (Rahman, Kode, Biswas, 2006). Reactive oxygen intermediates have been demonstrated to play an etiological role in myoglobinuric renal failure (Chander, Singh, Chopra, 2004). Reactive oxygen species (ROS) neutralized by GSH through a concerted cascade of detoxification mechanisms involving glutathione peroxidases (GPx), glutathione- S-transferases (GST), and glutathione reductase (GR). Apart from this, GSH also is involved in signal transduction, gene expression, apoptosis and host of other cellular reactions. Therefore, GSH/GSSG status is frequently measured in physiological and pathophysiological conditions (Rahman, Kode, Biswas, 2006).

Thus, it seemed interesting to look into the possible cure effect of MSM, which has not been investigated previously, on the effects of nephropathy in which free radicals appear to be involved.

\section{MATERIAL AND METHOD}

Wister rats weighing (250-300g) were adapted for one week before any experimental procedures. They fed with standard commercial rat pellets and allowed water ad libitum. They were kept under controlled environmental conditions (temperature $23 \pm 2{ }^{\circ} \mathrm{C}$, humidity $55 \pm 15 \%$, lighting regimen of 12-h light: 12-h dark ). All methods performed in this study were in accordance with regulatory guidance on the care and use of experimental animals.

\section{Experimental design and treatment protocol}

Three groups of rats ( $\mathrm{n}=6$ for each group), were employed in this study: group $1(\mathrm{~N})$, served as a control; group $2(\mathrm{G})$, was only given Glycerol (surechem products LTD) $(50 \%, 10 \mathrm{~mL} / \mathrm{kg}$, i.m.); group $3(\mathrm{M})$, was given Glycerol plus MSM (Panvo Organics Pvt Ltd) (400 mg/ $\mathrm{kg}$ ), starting after 60 mints of the glycerol injections.

After $24 \mathrm{~h}$ (hours) of water deprivation, on the first day, in groups $(\mathrm{G})$ and $(\mathrm{M})$, a kidney injury was induced in rats by intramuscular administration of glycerol $10 \mathrm{~mL} /$ $\mathrm{kg}(50 \%$ vol./vol. in saline) (Liu, Fu, Gou, 2013), half of the dose was administered in each hind limb muscle, at the injection of a single dose in the hind limb muscles of the rats. While the group $(\mathrm{N})$ had an equal volume of normal saline $(10 \mathrm{ml} / \mathrm{kg}$, i.m.). Drinking water and food, then resumed ad libitum.
After 60 minutes of glycerol injection, for consecutive 6 days, orally administered by using an intubation needle was done, by saline for group $(\mathrm{N})$, and (G); MSM (400 mg/kg) for the group (M) suspended with normal saline (Amirshahrokhi, Bohlooli, Chinifroush, 2011). An hour after the last dose, the animals were sacrificed under deep ethyl ether anesthesia (surechem products LTD). Blood samples and kidney tissues were harvested for future biochemical and pathology analyses.

\section{Renal function tests}

Blood samples were harvested by heart puncture. Serum was separated for renal function tests (serum urea and creatinine concentrations).

\section{Serum creatinine concentration}

Creatinine concentrations in plasma samples were measured with a rate-blanked and compensated picric acid colorimetric assay (CREA, Roche/Hitachi Modular $p$ analyzer). In this enzymatic method, creatinine is converted to creatine under the activity of creatininase. In alkaline solution, creatinine, forms a yellow-orange complex with picrate. The absorbance of samples and standard were measured twice (after $30 \mathrm{~s}$ and $90 \mathrm{~s}$ )) spectrophotometrically (Hitachi U-1800) at $505 \mathrm{~nm}$. The concentrations calculated according to the manufacturer's protocol.

\section{Serum urea concentration}

The principle of the urea measuring, using the Roche/Hitachi Modular $p$ analyzer kit, is based on the change in the intensity of staining ammonia compounds with sodium salicylate and sodium hypochlorite, which is directly proportional to the concentration of urea in the sample. The colored complex is measured by spectrophotometrically at $340 \mathrm{~nm}$, according to the manufacturer's protocol.

\section{Determination of reduced glutathione}

The nephrectomy was performed rapidly. The kidney was stored at $-80^{\circ} \mathrm{C}$ for biochemical determination of reduced glutathione (GSH). Glutathione Assay Kit (Abnova) for direct assay of reduced glutathione in kidney tissue was used. Tissue samples were washed in cold PBS. $150 \mathrm{mg}$ was measured and homogenized in 1-2 $\mathrm{mL}$ of cold phosphate buffer $(\mathrm{pH}=6-7)$ containing $1 \mathrm{mM}$ EDTA. The homogenate was centrifuged at $10000 \mathrm{~g}$ for 15 minutes at $4 \mathrm{c}$. The supernatant was stored at $-20^{\circ} \mathrm{C}$ for later use for GSH assay. The reduced glutathione (GSH) in the kidney tissue was measured spectrophotometrically 
according to the improved 5,5'-dithiobis (2-nitrobenzoic acid DTNB) method, which combined deproteination and detection (reagent A) into one reagent. DTNB reacts with reduced glutathione to form a yellow product. The optical density measured at $412 \mathrm{~nm}$ is directly proportional to the glutathione concentration in the sample.

\section{STATISTICAL ANALYSIS}

Results were expressed as (mean \pm standard Error SE). Statistical analysis was performed using the GraphPad Prism (version 6) statistical package. Comparisons between the groups for parametric were performed using a one-way analysis of variance (ANOVA) followed by Dunnett's multiple comparison tests, for serum creatinine, urea concentration, and reduced glutathione. Statistical significance was set at $(\mathrm{p}<0.0001)$.

\section{RESULTS}

\section{Effects of MSM on Kidney Function}

\section{Serum level of blood urea}

The serum levels of urea in $(\mathrm{G})$ rats were significantly higher than in $(\mathrm{N})$ rats $(\mathrm{P}<0.0001)$. The level of urea was $(33.85 \pm 9.237 \mathrm{mmol} / \mathrm{L})$ in $(\mathrm{N})$ rats, whereas the level of urea reached $(111.7 \pm 21.09 \mathrm{mmol} / \mathrm{L})$ in the $(\mathrm{G})$ rats. Treatment of animals with MSM significantly reduced the levels of urea $(51 \pm 6.261 \mathrm{mmol} / \mathrm{L})$ for the $(\mathrm{M})$ group compared with that of $(\mathrm{G})$ rats.

\section{Serum level of Serum creatinine $(\mathrm{Scr})$}

The level of serum creatinine was $(0.3983 \pm 0.08819$

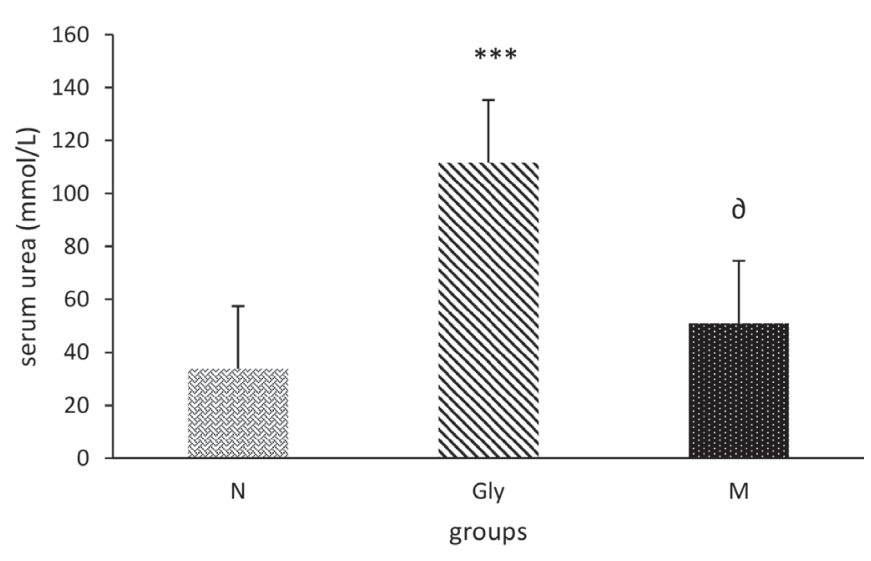

FIGURE 1 - Effects of MSM on serum urea level content in glycerol induced ARF. Each column is represented as the mean \pm SE. $* * * p<0.0001$ vs. $\mathrm{N}, \partial \mathrm{p}<0.0001$ vs. G. N, control group; GLY, Glycerol; M, MSM. $\mu \mathrm{mol} / \mathrm{L})$ in $(\mathrm{N})$ rats, whereas the level of Scr reached $(1.87$ $\pm 0.265 \mu \mathrm{mol} / \mathrm{L})$ in $(\mathrm{G})$ rats. Administration of MSM significantly reduced the levels of Scr $(0.628 \pm 0.0652$ $\mu \mathrm{mol} / \mathrm{L})$ for $(\mathrm{M})$ group compared with that of $(\mathrm{G})$ rats $(\mathrm{P}<0.0001)$.

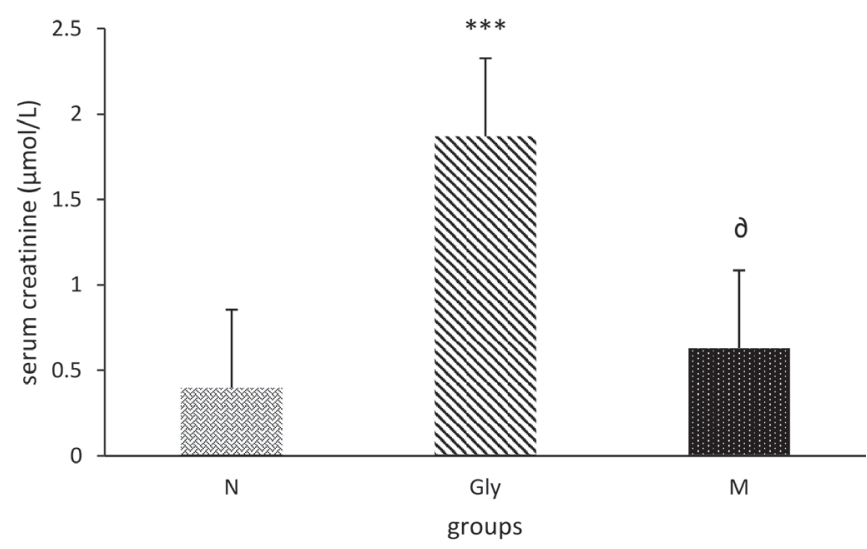

FIGURE 2 - Effects of MSM on serum creatinine level content in glycerol induced ARF. Each column is represented as the mean \pm SE. $* * * p<0.0001$ vs. N, $\partial \mathrm{p}<0.0001$ vs. G. N, control group; GLY, Glycerol; M, MSM.

\section{Reduced Glutathione levels}

The administration of glycerol significantly reduced the concentration of endogenous antioxidant glutathione as compared to control group. Treatment of animals with MSM significantly increased the glutathione concentration compared to $(\mathrm{G})$ group $(\mathrm{P}<0.0001)$.

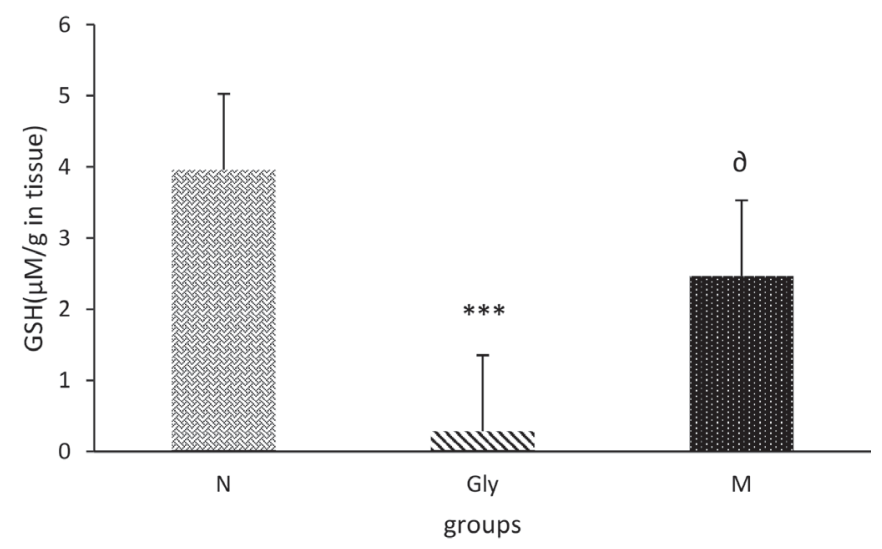

FIGURE 3 - Effects of MSM on renal glutathione (GSH) content in glycerol induced ARF. Each column is represented as the mean \pm SE. $* * * p<0.0001$ vs. N, $\partial \mathrm{p}<0.0001$ vs. G.

\section{DISCUSSION}

The most commonly used model for studying 
ARF is a rat receiving a single intramuscular injection of glycerol, which induces rhabdomyolysis. A number of studies have shown that rhabdomyolysis-induced myoglobinuric ARF (Park et al., 2012). Glycerol-induced renal failure closely mimics the rhabdomyolysis; schemiareperfusion-induced acute renal failure (ARF) in humans, simulates the hemodynamic changes-induced change in renal functioning. The pathogenesis of glycerol-induced myoglobinuric acute renal failure involves ischemia, vascular congestion and reactive oxygen metabolites (Manikandan et al., 2014), and tubular necrosis (Singh et al., 2012). Renal injury associated with ischemia/ reperfusion results from a dynamic process involving the vasculature and tubules in a complex interaction, whereby events modulating the vasculature will alter oxygen and nutrient delivery to the epithelial cell. So the injured epithelial cell will respond by producing autocrine factors that will affect its own survival and paracrine factors, which affect the vasculature (Bonventre, Weinberg, 2003).

The results of this study show that glycerol administration produced a typical pattern of nephrotoxicity, which manifested by an increased level of serum levels of urea and creatinine; they were increased significantly in the $(\mathrm{G})$ rats by $230 \%$ and $369 \%$, respectively, compared with $(\mathrm{N})$ rats. In contrast, it reduced significantly by $54 \%$ and $66 \%$, respectively, compared with $(\mathrm{G})$ rats. The results were in agreement with many studies such as Stefanovic (Stefanovic et al., 2000), and Manikandan et al. (2014). However, MSM administration showed a significant decrease in their levels.

There are some experimental data suggesting that nephrotoxic drugs can alter the levels of kidney markers, glutathione, and other antioxidant enzymes, which are commonly used to monitor the development and extent of renal tubular damage due to oxidative stress (Manikandan et al., 2014). Glutathione is an important intracellular agent, which protects the cell from the damage caused by free radicals. In addition, Kidney has an important role in GSH metabolism (Tiodorović, Cvetković, 2004).

Reactive oxygen and nitrogen species are known to exert profound, and often opposing, effects in inflamed tissues. These actions can be manifested as impaired endothelium-dependent vasodilatation, activation of nuclear transcription factors, and the subsequent production of inflammatory cytokines, enhanced recruitment and activation of leukocytes, accelerated apoptosis, and parenchymal cell necrosis (Di Paola et al., 2005). Oxidative stress decreases the enzyme activities. This situation would lead generating the free radicals, which would induce GSH depletion, thus increasing oxidative stress that would reduce antioxidant enzyme levels (Marañón et al., 2008). Likewise, the results of the present study, which manifested by a significantly decreased level of GSH in the (G) rats by $93 \%$ compared with $(\mathrm{N})$ rats. That was in fair agreement with a prior observation (Manikandan et al., 2014), that added the oxidative changes induced by intraperitoneal glycerol injection reported by Rieger et al. (2008). In contrast, it increased significantly by $759 \%$ in the $(\mathrm{M})$ rats compared with $(G)$ rats.

Methionine, an essential amino acid, and cysteine are the major sulfur-containing amino acids in the body, and both synthesized predominantly in plants and microorganisms. MSM is a natural constituent of the environment which is a part of the natural global sulfur cycle (Richmond, 1986). MSM is a naturally occurring sulphur compound that MSM may play a role in the synthesis of glutathione (GSH), one of the most important intracellular antioxidants, through its trans-sulfuration pathway (Marañón et al., 2008); equally Group (M) induced an increase in GSH levels. Our findings are in agreement with previous reports, that studying the protective effect on pulmonary arterial hypertension, where Mohammadi showed that MSM could exert protective anti-oxidative effects through the induction of serum CAT, SOD, and glutathione peroxidase (GSH-Px) activities along with associated reducing agents, such as increasing GSH (Mohammadi et al., 2012; Amirshahrokhi, Bohlooli, Chinifroush, 2011). The results of numerous studies confirm the importance of GSH esters in the prevention and in the medical treatment of acute renal failure. The protective effect of GSH may be due to either increased intracellular GSH levels or the presence of GSH in tubular fluid, or both (Tiodorović, Cvetković, 2004).

MSM is known as an antioxidant, which can scavenge ROS, thus preventing tissue damage. It has also shown an exertion of protective effects on different experimental pathological situations, in which free radicals and ROS are involved (Marañón et al., 2008). So the ability of MSM to mitigate glycerol-induced nephropathy is partly due to its serving action as a sulfur donating agent for the synthesis of new cysteine, a rate-limiting precursor of GSH production (Marañón et al., 2008).

The results of the present study demonstrated that treatment of glycerol-induced ARF in rats with MSM markedly improved their renal injury. Moreover, all parameters indicating the presence of oxidative injury were inhibited by the MSM, suggesting that MSM effect is probably due to the antioxidant activity. This may provide therapeutic opportunities of treating myoglobinuric ARF in humans. Concerning our knowledge, this is the first study that shows MSM may heal ARF. 


\section{CONCLUSIONS}

Based on the data given in this study, it can be confirmed that a dose of $400 \mathrm{mg}$ of MSM, has cured the effects of acute experimental ARF.

\section{REFERENCES}

Amirshahrokhi K, Bohlooli S, Chinifroush M. The effect of methylsulfonylmethane on the experimental colitis in the rat. Toxicol Appl Pharm. 2011;253(3):197-202.

Bohlooli S, Mohammadi S, Amirshahrokhi K, Mirzanejad-ASL H, Yosefi M, Mohammadi-NEI A, Chinifroush MM. Effect of methylsulfonylmethane pretreatment on aceta-minophen induced hepatotoxicity in rats. Iran J Basic Med Sci. 2013;16(8): 896-900.

Bonventre JV, Weinberg JM. Recent advances in the pathophysiology of ischemic acute renal failure. J Am Soc Nephrol. 2003;14(8):2199-2210.

Chander V, Singh D, Chopra K. Reversal of experimental myoglobinuric acute renal failure in rats by quercetin, a bioflavonoid. Pharmacology. 2004;73(1):49-56.

Childs SJ. Dimethyl sulfone (DMSO2) in the treatment of interstitial cystitis. Urol Clin North Am. 1994;21(1):85-88.

Di Paola R, Mazzon E, Patel NS, Genovese T, Muià C, Thiemermann C, De Sarro A, Cuzzocrea S. Beneficial effects of GW274150 treatment on the development of experimental colitis induced by dinitrobenzene sulfonic acid. Eur J Pharmacol. 2005;507(1-3):281-289.

Horvath K, Noker P, Somfai-Relle S, Glavits R, Financsek I, Schauss A. Toxicity of methylsulfonylmethane in rats. Food Chem Toxicol. 2002;40(10):1459-1462.

Kim L, Axelrod L, Howard P, Buratovich N, Waters R. Efficacy of methylsulfonylmethane (MSM) in osteoarthritis pain of the knee: a pilot clinical trial. Osteoarthr Cartilage. 2006;14(3):286294.

Liu Y, Fu X, Gou L, Li S, Lan N, Zheng Y, Yin X. L-citrulline protects against glycerol-induced acute renal failure in rats. Renal Failure. 2013;35(3):367-373.
Manikandan R, Beulaja M, Thiagarajan R, Pandi M, Arulvasu C, Prabhu NM, Saravanan R, Esakkirajan M, Palanisamy S, Dhanasekaran G. Ameliorative effect of ferulic acid against renal injuries mediated by nuclear factor-kappaB during glycerol-induced nephrotoxicity in Wistar rats. Renal Failure. 2014;36(2):154-165.

Marañón G, Muñoz-Escassi B, Manley W, Garcia C, Cayado P, De La Muela MS, Olábarri B, León R, Vara E. The effect of methyl sulphonyl methane supplementation on biomarkers of oxidative stress in sport horses following jumping exercise. Acta Vet Scand. 2008;50:45.

Mohammadi S, Najafi M, Hamzeiy H, Maleki-Dizaji N, Pezeshkian M, Sadeghi-Bazargani H, Darabi M, Mostafalou S, Bohlooli S, Garjani A. Protective effects of methylsulfonylmethane on hemodynamics and oxidative stress in monocrotaline-induced pulmonary hypertensive rats. Adv Phar Sci. 2012;2012:507278.

Parcell S. Sulfur in human nutrition and applications in medicine. Altern Med Rev. 2002;7(1):22-44.

Park CH, Tanaka T, Cho EJ, Park JC, Shibahara N, Yokozawa T. Glycerol-induced renal damage improved by 7-O-galloyl-Dsedoheptulose treatment through attenuating oxidative stress. Biol Pharma Bull. 2012;35(1):34-41.

Rahman I, Kode A, Biswas SK. Assay for quantitative determination of glutathione and glutathione disulfide levels using enzymatic recycling method. Nat Protoc. 2006;1(6):31593165 .

Richmond VL. Incorporation of methylsulfonylmethane sulfur into guinea pig serum proteins. Life Sci. 1986;39(3):263-268.

Rieger E, Rech VC, Feksa LR, Wannmacher C. Intraperitoneal glycerol induces oxidative stress in rat kidney. Clin Exp Pharmacol Physiol. 2008;35(8):928-933.

Singh AP, Muthuraman A, Jaggi AS, Singh N, Grover K, Dhawan R. Animal models of acute renal failure. Pharmacol Rep. 2012;64(1):31-44.

Stefanovic V, Savic V, Vlahovic P, Cvetkovic T, Najman S, Mitic-Zlatkovic M. Reversal of experimental myoglobinuric acute renal failure with bioflavonoids from seeds of grape. Renal Failure. 2000;22(3):255-266. 
Tiodorović $\mathrm{D}$, Cvetković T. Gluthatione content in blood and kidney in glycerol induced acute renal failure. 2004;21(2):85-88.

Vanholder R, Sever MS, Erek E, Lameire N. Rhabdomyolysis. J Am Soc Nephrol. 2000;11(8):1553-1561.
Zager RA. Rhabdomyolysis and myohemoglobinuric acute renal failure. Kidney Int. 1996;49(2):314-326.

Received for publication on $12^{\text {th }}$ August 2017 Accepted for publication on $20^{\text {th }}$ October 2017 